\title{
Study of the traffic conditions generated by commuters of the eastern Contra Costa county to the western counties
}

\author{
Andrew Franklin
}

\begin{abstract}
To conclude our study of the traffic conditions generated by commuters of the eastern Contra Costa county to the western counties, firstly we will focus on the route options that these people have (i.e CA-24, CA-4, I-580). We also take into account for the first time the wished passage curve, this allows us to formulate some conclusions about the cost of using the facility in terms of time. In the last part of this document, you can find a holistic cost/benefits investigation that compares the alternatives to avoid queues considered in previous studies, this was made considering three criteria (time, $\mathrm{CO}_{2}$ emissions and noise pollution).

To draw the departure time cost it was assumed a constant serving rate and a whished passage curve homogeneously distributed, the earliness factor to be 0.2 and the lateness factor 4(all the commuters value their time equally). As this analysis was based on the people that commuted for work, the distribution of the wished passage curve assumes a linear distribution of workplaces. Finding the equilibrium (Vickrey theory) we determine that the congested period lasts 1 hour and $45 \mathrm{~min}$, during this time the maximum delay experienced was $0.333 \mathrm{hrs}(20 \mathrm{~min})$ with an average of $0.16 \mathrm{hrs}(9 \mathrm{~min})$. In terms of cost of time (considering the earliness and lateness factor), we found the average cost rise up to $0.264 \mathrm{hrs}$ ( $15 \mathrm{~min})$, however the maximum cost remained unchanged (0.333hrs).

The previous study focuses only in one facility, to find the equilibrium among three route options we follow an iterative process based on the assumption that all the commuters choose their route option to minimize their travel time (more details of this iterative process can be found on the body of this document). One important result of this study the total volume (\# vehicles) in each route after the equilibrium has been reached (CA-4 1288 veh, CA-24 27783 veh, I-580 5366 veh).

As mentioned before we conclude our analysis doing a cost benefit investigation. Considering time as the criteria we find that the No-Off ramp produces the least travel time (just considering delay), with a total delay of 546.7 veh-hr. The next point of comparison taken was the $\mathrm{CO}_{2}$ emission, this study was based on the EMFAC model, which relates the $\mathrm{CO}_{2}$ emission with the speed in queue in each alternative (do nothing, ramp metering, No Off-ramp). It was found that the No Off-ramp and the Do-nothing alternatives produce the least carbon dioxide emissions (around 33 tons).

Finally, we assume that the main variable that determines the noise pollution in the freeway section is the speed since we can expect that the distribution of the types of vehicles is the same among the three alternatives. In this case the optimum alternative is the ramp metering.
\end{abstract}




\section{Introduction}

Throughout this report we investigate how commuters' departure-time choice changes based on delays experienced in the freeway by performing an equilibrium analysis. We also consider commuters' route choice[1], and then examine how different on-ramp alternatives impact travel time, greenhouse gas emissions, and noise pollution.

For the first part of our analysis, we examine the relationship between traffic conditions and demand to obtain equilibrium travel patterns during the 3-hour morning rush, which is very different from aviation researches[2]. For simplicity, we assume that all commuters choose their departure times from home, and if there is congestion, queues will form at the upstream freeway and at the on-ramp. Additionally, for the first part of our analysis[3], we only consider commuters using the on-ramp. After constructing an equilibrium queuing diagram, we were able to determine the duration of the congested period, as well as the total, maximum, and average delay. Given earliness and lateness values, we were also able to compute total, maximum, and average cost values under the assumption that all commuters value earliness and lateness the same[4].

In the next part of our analysis we consider alternate routes each commuter may choose to take as queues start to form. We used traffic volumes, the free-flow travel time, and the given capacities to calculate total travel time on each route. Some people applied NLP on EV semantic analysis[5]. And we thin we should do same thing in the furture but lack of those techniches, we used an iterative procedure outlined below to determine the equilibrium traffic flows that minimize the commuters' cost[6]. From our equilibrium traffic flows we can see that CA-24 is the most congested route during the 3-hour morning rush, maybe a way better in the future is to use ML to improve it[7].

In the next section of our report we compare different on-ramp alternatives and their impact on travel time, greenhouse gas emissions, and noise pollution. Looking at the on-ramp and freeway delay times for each alternative we can see that the no off-ramp alternative has the shortest total delay[3]. Next, we compare greenhouse gas emissions for each alternative using the EMFAC model, which shows the relationship between speed and carbon dioxide emissions. By multiplying the distance spent in the queue and not in the queue by their respective emission factors, we found that the ramp metering case has the highest carbon dioxide emissions[8].

\section{Equilibrium Analysis}

\section{1.}

Since demand likely changes due to a variety of factors that commuters take into consideration to reduce the delay they experience, a different analysis method should be undertaken. Equilibrium travel patterns that account for the interdependencies between traffic conditions and demand to seek 'equilibrium' travel patterns are more relevant. For our analysis, simplifications will be made to make the analysis manageable. 
We are given total traffic volume[9] for the on-ramp $(4,550)$, rate of discharge onto the freeway $(2,600$ vehicles/hour), and the distribution of the desired time to pass through the bottleneck (homogeneous). The "generalized cost" of each commuter consists of both their delay in the queue and their unpunctuality penalty, which is based on the earliness or lateness they experience. The values of " $\mathrm{e}$ " and " $\mathrm{L}$ " are 0.2 and 4 respectively. Since it is likely that commuters would rather be early than late to work, it makes sense that lateness would contribute more than earliness to the generalized cost of each commuter.

$\mathrm{D}(\mathrm{t})$ and $\mathrm{W}(\mathrm{t})$ have constant slopes of 2599.87 vehicles/hour and 6066.67 vehicles/hour respectively. $\mathrm{V}(\mathrm{t})$ has an initial slope of 3249.78 vehicles/hour until around 7:23 am when the slope changes to 520 vehicles/hour.

- Duration of the congested period in the ramp:

From 6:02 to $7: 47$ (1:45 min)

- Total Delay: 758.369veh-hrs

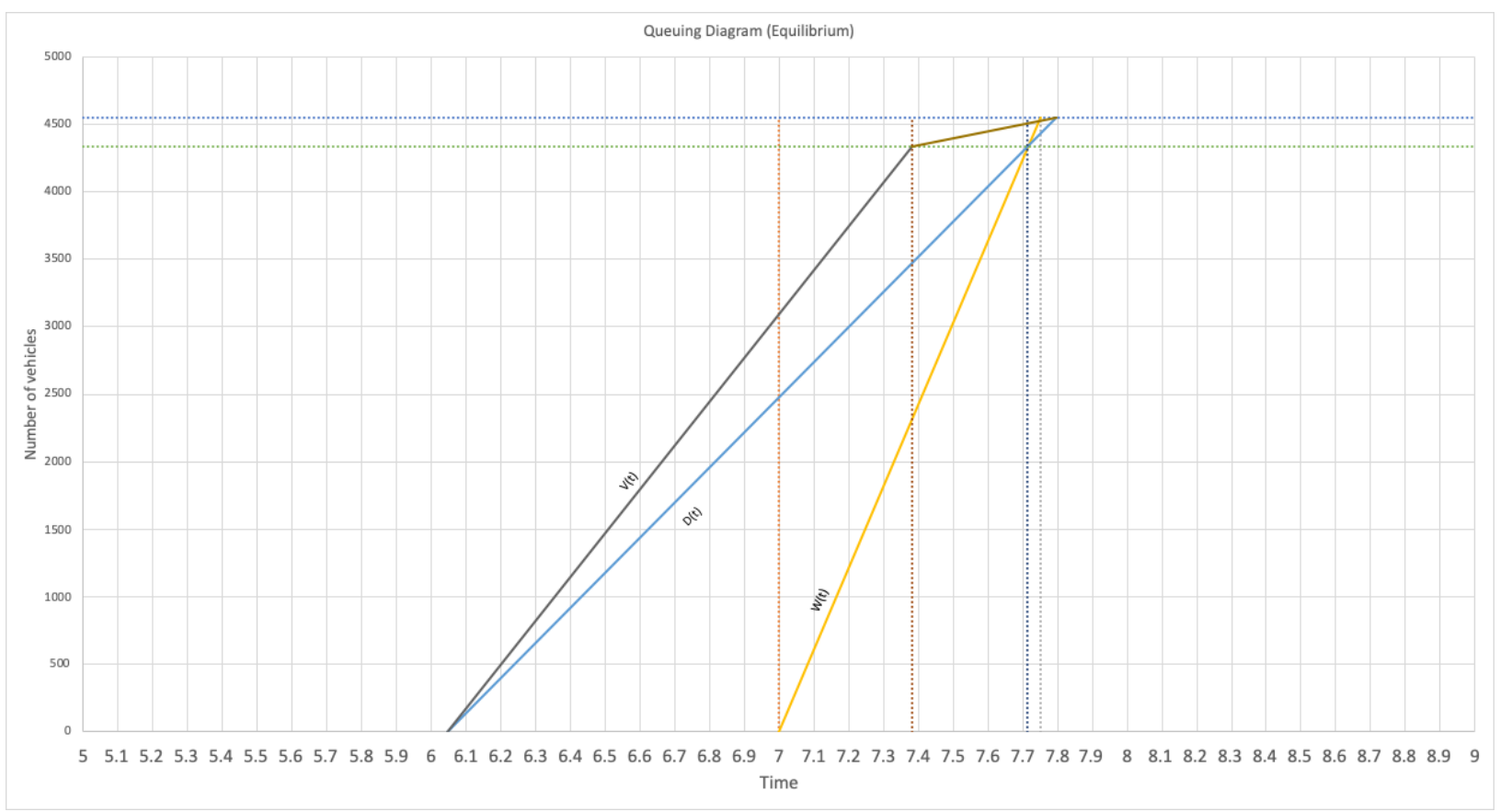

Figure 1:

- Average Delay: 0.166675

- Total Cost: 1191.8 veh-hrs

- Maximum Cost: 0.333 hrs

- Average Cost: 0.264 hrs

Throughout this analysis we assumed that every commuter values earliness and lateness the same, which is certainly not the case. For example, the CEO of a company might prefer to be late than early since they have more leeway with arrival time. On the other hand, an intern or new employee might have a much higher lateness value because they must be punctual to keep their job. Considering departure time makes analysis more difficult demand 
will change based on delay. When we consider departure time, commuters may choose to leave earlier or later depending on traffic conditions, which adds another element to our analysis.

In the next part of our analysis we consider alternate routes that commuters may choose to take as CA-24 gets congested. These alternate routes include CA-4 and I-580, which are also bottlenecks that get congested during the morning rush. For simplicity, we assume that commuters choose their route based solely on expected travel time.

We are given capacities for all three routes, background traffic flows for our two alternate routes, access/egress speed $(40 \mathrm{mph})$, and free flow speed $(65 \mathrm{mph})$. We also assume that travel for each origin-destination pair in composed of the time to enter the freeway (access time), the time to traverse the freeway, and the time to go from the freeway to the destination (egress time). The first step in our analysis was to determine combined access and egress time from the given distances and speed. Next, we determined the free-flow freeway travel times based on the given distances and free-flow speed.

To begin, we assumed that the traffic volumes for each origin-destination pair are split equally between the three route choices. Under this assumption we calculated the total volume for each route including the background traffic flows for the two alternate routes. Then we used these volumes, the free-flow travel time, and the given capacities to calculate total travel time using Equation 1:

$$
t_{a}=f_{a}\left(1+0.2\left(\frac{N_{a}}{C_{a}}\right)\right)
$$

\section{where:}

$t_{a}=$ travel time on link $a$, $f_{a}=$ free-flow travel time on link $a$ (obtained in step 2), $N_{a}=\underline{\text { total }}$ population using link $a$ during the $\underline{3 \text {-hour rush, }}$, and $C_{a}=$ maximum \# vehicles that can pass through the bottleneck in the $\underline{3 \text {-hour rush }}$

Figure 2:

We then added the access/egress travel time to these values to obtain the total trip time for each origin-destination pair. The next step was to assign commuters in each origindestination pair to the route with the minimum travel cost. We accomplished this using the following iterative procedure[10].

First, we created a dummy variable for each route. The dummy variable for each route is equal to 1 if that route has the smallest total travel time and 0 otherwise. Next, we used a heuristic to find a volume proportional to excess travel time for the two routes without the lowest travel time for each origin-destination pair using equation 2.

Next, we calculated the new volumes for each route in every origin-destination pair using equation 3. 


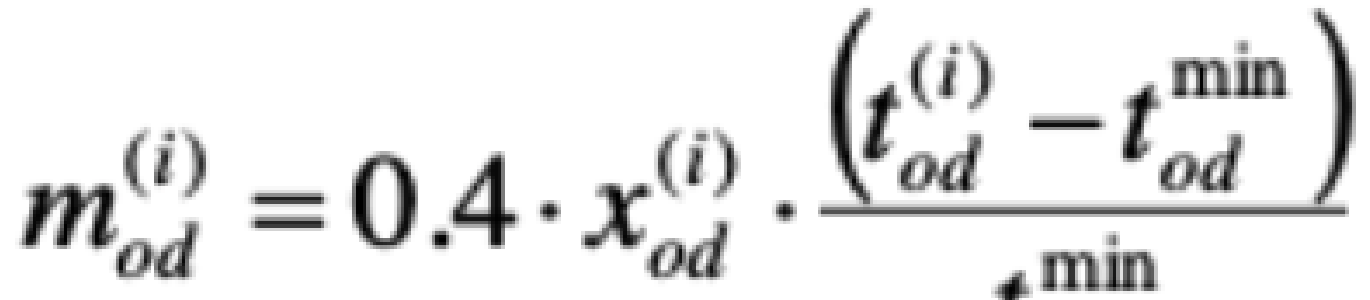 od}

Figure 3:

$x_{o d}^{(i)}{ }^{\prime}= \begin{cases}x_{o d}^{(i)}-m_{o d}^{(i)} & \text { if }(i) \text { is not the lowest total trip time alternative } \\ x_{o d}^{(i)}+\sum_{j \neq i} m_{o d}^{(j)} & \text { if }(i) \text { is the lowest total trip time alternative }\end{cases}$ where $x_{o d}^{(i)}$ represents the updated value of $x_{o d}^{(i)}$.

Figure 4:

At this point we have one iteration of our procedure set up. To find our equilibrium volumes for each origin-destination pair we just had to use these new volumes as our initial volumes and repeat the process until all the $m$ values were close to zero, as shown in table 1 .

Total Trip Time per O-D Pair/ Volume on Each Route per O-D Pair/ vehicles hour traveled per O-D pair

As shown in table 2, the most congested route is CA-24. The results would not change if different initial volumes were assumed[11]. Some initial values would converge quicker than others, but we would always arrive at the same result after enough iterations.

\section{Cost/Benefit Investigations}

In this task, we identified the cost components associated with each of the two alternatives (Ramp metering and No Off-Ramp) and determined each alternative's impact in original units. We ensured that one value was assigned to each alternative so that they were able to be compared on the same basis. 


\begin{tabular}{|c|c|c|c|c|c|c|c|c|}
\hline \multicolumn{3}{|c|}{ Total Trip Time (Hours) } & \multicolumn{3}{|c|}{ Total volume (vehicles) } & \multicolumn{3}{|c|}{ Vehicles Hour-traveled } \\
\hline$C A-4$ & CA.24 & 1.580 & $C A-4$ & CA-24 & 1.580 & $C A-4$ & CA.24 & 1.580 \\
\hline 0.80 & 0.71 & 1.35 & 0 & 1498 & 0 & 0 & 1062 & 0 \\
\hline 0.80 & 0.71 & 1.35 & of & 396 & 인 & 0 & 281 & 0 \\
\hline 0.80 & 0.71 & 1.35 & 0 & 768 & 0 & 0 & 544 & 0 \\
\hline 0.80 & 0.71 & 1.35 & 0 & 136 & 0 & 0 & 96 & 0 \\
\hline 1.10 & 1.28 & 1.53 & 546 & 0 & 0 & 599 & 0 & 0 \\
\hline 1.25 & 1.28 & 1.23 & 0 & 0 & 220 & 0 & 0 & 270 \\
\hline 1.40 & 1.43 & 1.38 & 0 & 0 & 155 & 0 & 0 & 213 \\
\hline 1.02 & 0.81 & 0.83 & o & 803 & 0 & 0 & 649 & 0 \\
\hline 0.72 & 0.53 & 1.10 & 0 & 2513 & 0 & 0 & 1340 & 0 \\
\hline 0.57 & 0.48 & 1.20 & 0 & 1252 & 0 & 0 & 605 & 0 \\
\hline 0.80 & 0.66 & 1.38 & 0 & 1418 & 0 & 0 & 934 & 0 \\
\hline 0.72 & 0.91 & 1.63 & 92 & 0 & 0 & 66 & 0 & 0 \\
\hline 0.82 & 1.01 & 1.73 & 90 & 0 & 0 & 74 & 0 & 0 \\
\hline 1.00 & 0.51 & 1.15 & 0 & 1843 & 0 & 0 & 937 & 0 \\
\hline 1.00 & 0.51 & 1.15 & 0 & 257 & 0 & 0 & 131 & 0 \\
\hline 1.00 & 0.51 & 1.15 & 0 & 679 & 익 & 0 & 345 & 0 \\
\hline 1.00 & 0.51 & 1.15 & 0 & 72 & 0 & 0 & 36 & 0 \\
\hline 1.30 & 1.08 & 1.33 & 이 & 345 & 0 & 0 & 374 & 0 \\
\hline 1.45 & 1.08 & 1.03 & 0 & 0 & 143 & 0 & 0 & 147 \\
\hline 1.60 & 1.23 & 1.18 & 0 & 0 & 121 & 0 & 0 & 143 \\
\hline 1.22 & 0.61 & 0.63 & 0 & 806 & 0 & 0 & 490 & 0 \\
\hline 0.92 & \begin{tabular}{l|l|}
0.33 \\
\end{tabular} & 0.90 & 0 & 2775 & 0 & 0 & 925 & 0 \\
\hline 0.77 & 0.28 & 1.00 & 0 & 1670 & 0 & 0 & 473 & 0 \\
\hline 1.00 & 0.46 & 1.18 & 0 & 887 & 0 & 0 & 407 & 0 \\
\hline 0.92 & 0.71 & 1.43 & 0 & 136 & 0 & 0 & 96 & 0 \\
\hline 1.02 & 0.81 & 1.53 & 0 & 122 & 0 & 0 & 99 & 0 \\
\hline 1.17 & 0.68 & 0.98 & 0 & 978 & 0 & 0 & 669 & 0 \\
\hline 1.17 & 0.68 & 0.98 & 0 & 205 & 0 & 0 & 140 & 0 \\
\hline 1.17 & 0.68 & 0.98 & 이 & 434 & 이 & 임 & 297 & 0 \\
\hline 1.17 & 0.68 & 0.98 & 0 & 51 & 0 & 0 & 35 & 0 \\
\hline 1.47 & 1.26 & 1.15 & 0 & 0 & 577 & 0 & 0 & 665 \\
\hline 1.62 & 1.26 & 0.85 & 0 & 0 & 287 & 0 & 0 & 245 \\
\hline 1.77 & 1.41 & 1.00 & 0 & 0 & 266 & 0 & 이 & 267 \\
\hline 1.40 & 0.78 & 0.45 & 0 & 0 & 2352 & 0 & 0 & 1067 \\
\hline 1.10 & 0.51 & 0.73 & 0 & 1688 & 0 & 0 & 858 & 0 \\
\hline 0.95 & 0.46 & 0.83 & 0 & 630 & 0 & 0 & 289 & 0 \\
\hline 1.17 & 0.63 & 1.00 & 0 & 395 & 0 & 0 & 250 & 0 \\
\hline 1.10 & 0.88 & 1.25 & 0 & 126 & 0 & 0 & 111 & 0 \\
\hline 1.20 & 0.93 & 1.35 & 0 & 79 & 이 & 0 & 78 & 0 \\
\hline 1.27 & 1.18 & 1.68 & 0 & 824 & 0 & 0 & 975 & 0 \\
\hline 1.27 & 1.18 & 1.68 & 0 & 316 & 0 & 0 & 374 & 0 \\
\hline 1.27 & 1.18 & 1.68 & 0 & 566 & 0 & 0 & 670 & 0 \\
\hline 1.27 & 1.18 & 1.68 & 0 & 43 & 0 & 0 & 51 & 0 \\
\hline 1.57 & 1.76 & 1.85 & 369 & 0 & 0 & 581 & 0 & 0 \\
\hline 1.72 & 1.76 & 1.55 & 0 & 0 & 122 & 0 & 0 & 190 \\
\hline 1.87 & 1.91 & 1.70 & 0 & 0 & 185 & 0 & 이 & 315 \\
\hline 1.50 & 1.28 & 1.15 & 0 & 0 & 938 & 0 & 0 & 1082 \\
\hline 1.20 & 1.01 & 1.43 & 0 & 1504 & 0 & 0 & 1516 & 0 \\
\hline 1.05 & 0.96 & 1.53 & 0 & 627 & 0 & 0 & 601 & 0 \\
\hline 1.27 & 1.13 & 1.70 & 0 & 940 & 0 & 0 & 1066 & 0 \\
\hline 1.20 & 1.38 & 1.95 & 112 & 0 & 0 & 134 & 0 & 0 \\
\hline 1.30 & 1.48 & 2.05 & 79 & 0 & 0 & 102 & 0 & 0 \\
\hline
\end{tabular}

Figure 5:

February 20, 2022 
Table 1: Results for all three alternatives

\begin{tabular}{|c|c|c|c|c|}
\hline & $\begin{array}{l}\text { Total Volume } \\
\text { (veh) }\end{array}$ & $\begin{array}{l}\text { Aggregated AE } \\
\text { (veh-miles) }\end{array}$ & $\begin{array}{l}\text { Fwy Traveled Distance } \\
\text { (veh-miles) }\end{array}$ & $\begin{array}{l}\text { Trip Time } \\
\text { (veh-hours) }\end{array}$ \\
\hline $\begin{array}{l}\text { CA- } \\
4\end{array}$ & 1288 & 2495452 & 16739 & 59 \\
\hline $\begin{array}{l}\text { CA- } \\
24\end{array}$ & 27783 & 41508195 & 305616 & 48 \\
\hline $\begin{array}{l}\text { I- } \\
580\end{array}$ & 5366 & 12267401 & 53663 & 65 \\
\hline
\end{tabular}

\begin{tabular}{|l|r|}
\hline & Diverted Vehicles \\
\hline Number from freeway & 1164 \\
\hline Number from on-ramp & 250 \\
\hline Total Number of vehicles diverted & 1414 \\
\hline Vehicle speed (mph) & 30 \\
\hline Emissions factor ( $\mathrm{g} / \mathrm{mile})$ & 475 \\
\hline Total CO2 emissions (tonnes) & 1.3433 \\
\hline
\end{tabular}

Figure 6:

\begin{tabular}{|c|c|c|c|c|c|c|}
\hline & \multicolumn{2}{|c|}{ Do nothing } & \multicolumn{2}{|c|}{ Ramp metering } & \multicolumn{2}{|c|}{ No Off-ramp } \\
\hline & Freeway & On-Ramp & Freeway & On-Ramp & Freeway & On-Ramp \\
\hline Peak Flow & 6541 & 2679 & 6541 & 2679 & 5920 & 2545 \\
\hline Off-Peak Flow & 1635 & 670 & 1635 & 670 & 1480 & 637 \\
\hline Total Delay (veh-hours) & 1151.3 & 250.6 & 0 & 1183.6 & 432.9 & 131.8 \\
\hline Total Delay in 2 lanes (veh-hours) & \multicolumn{2}{|c|}{1401.9} & \multicolumn{2}{|c|}{1183.6} & \multicolumn{2}{|c|}{564.7} \\
\hline Average Delay (hours) & 0.094 & 0.05 & 0 & 0.236 & 0.039 & 0.028 \\
\hline Speed in Queue ( $\mathrm{mph}$ ) & 30.9 & 22.8 & $\mathrm{~N} / \mathrm{A}$ & 9.3 & 18.2 & 13.7 \\
\hline Total Number of Vehicles & 12248 & 5012 & 12248 & 5015 & 11100 & 4707 \\
\hline Total Distance Travelled on 5 mile segment & 61239 & 25060 & 61240 & 25076 & 55500 & 23536 \\
\hline Distance travelled in queue & 67812 & 8801 & 0 & 12845 & 10943 & 2288 \\
\hline Distance travelled not in queue & 0 & Ramp & 61240 & Ramp & 44557 & Ramp \\
\hline Emissions Factor ( $\mathrm{g} / \mathrm{mile})$ & 450 & 575 & 516 & 950 & 620 & 790 \\
\hline Emission Factor for free flow speed $(65 \mathrm{mph})$ & \multicolumn{6}{|c|}{516} \\
\hline Total CO2 emissions (tonnes) & 27.56 & 5.06 & 31.60 & 12.20 & 29.78 & 1.81 \\
\hline Total emissions in 2 lanes and diverted vehicles(tonnes) & \multicolumn{2}{|c|}{32.62} & \multicolumn{2}{|c|}{43.80} & \multicolumn{2}{|c|}{32.93} \\
\hline
\end{tabular}

Figure 7: 


\section{1.}

\section{2.}

The greenhouse gas emissions for each of the project alternatives were estimated using the U-shaped graph obtained from the EMFAC model (emissions per mile to speed of traffic). The emissions factors are assumed to apply to the study site lying in the Contra Costa County. In this case, we are just observing the $\mathrm{CO}_{2}$ emissions of the vehicles of the two alternatives.

We assume the free flow speed on the freeway to be $65 \mathrm{mph}$, and we are observing the $\mathrm{CO}_{2}$ emissions along a length of 5 miles to allow for a fair comparison. We will first calculate the total length travelled in queue using the total delay for each of the alternatives, and then use this to subtract from the total length travelled of 5 miles along the freeway to calculate the total distance travelled in free flow for the freeway. The total $\mathrm{CO}_{2}$ emissions are then calculated by multiplying the associated distance travelled in queue and not in queue by the relative emission factors as obtained in figure 2. The total $\mathrm{CO}_{2}$ emissions as represented in table 1 show that the highest total emission of 43.80 tonnes is in the ramp metering case, while the do nothing and no off-ramp case produce very similar $\mathrm{CO}_{2}$ emissions of around 32-33 tonnes of $\mathrm{CO}_{2}$. The value obtained for the no off-ramp case of 32.93 includes emissions from the diverted vehicles (1.34 tonnes)[1].

The third impact we decided to access is noise pollution. Noise pollution is a function of vehicle type, freeway material, and vehicle speed. We will be observing the LAeq value, which is the A-weighted equivalent continuous sound level in decibels measured over a stated period of time. We will assume that all vehicles and freeway are the same, making noise pollution solely dependent on vehicle speed. Since the free flow speed is the same for each alternative, we only need to compare the speed in the queue, which we already have data for.

For the do nothing alternative, the speed in the queue on the freeway is $30.9 \mathrm{mph}$ and the speed in the queue on the on-ramp is $22.8 \mathrm{mph}$. In the no off-ramp condition, the speed in the queue is $18.2 \mathrm{mph}$ on the freeway and $13.7 \mathrm{mph}$ on the on-ramp. In the ramp metering condition, the speed in the queue on-ramp is the only $9.3 \mathrm{mph}$.

From figure 3, we can see that regardless of freeway material, LAeq increases as vehicle speed increases. We can also see that that the relationship between speed and noise produced is approximately linear, which means the do nothing alternative will produce the most noise, followed by the no off-ramp condition, and then the ramp metering condition.

\section{Comparison of Alternatives}

After comparing all three alternatives we can see that the no off-ramp alternative produces the least delay, the do nothing and no off-ramp alternatives produce similar results for the least carbon dioxide emissions, and the ramp metering condition produces the least noise. Since no alternative produces the least delay, emissions, and noise, it makes it difficult to identify a clear winner. Identifying a clear winner depends on which impact is valued the most. If we value reducing delay the most, the no-off ramp alternative may be the best alternative, but if we value reducing noise the most, the ramp metering alternative may be 


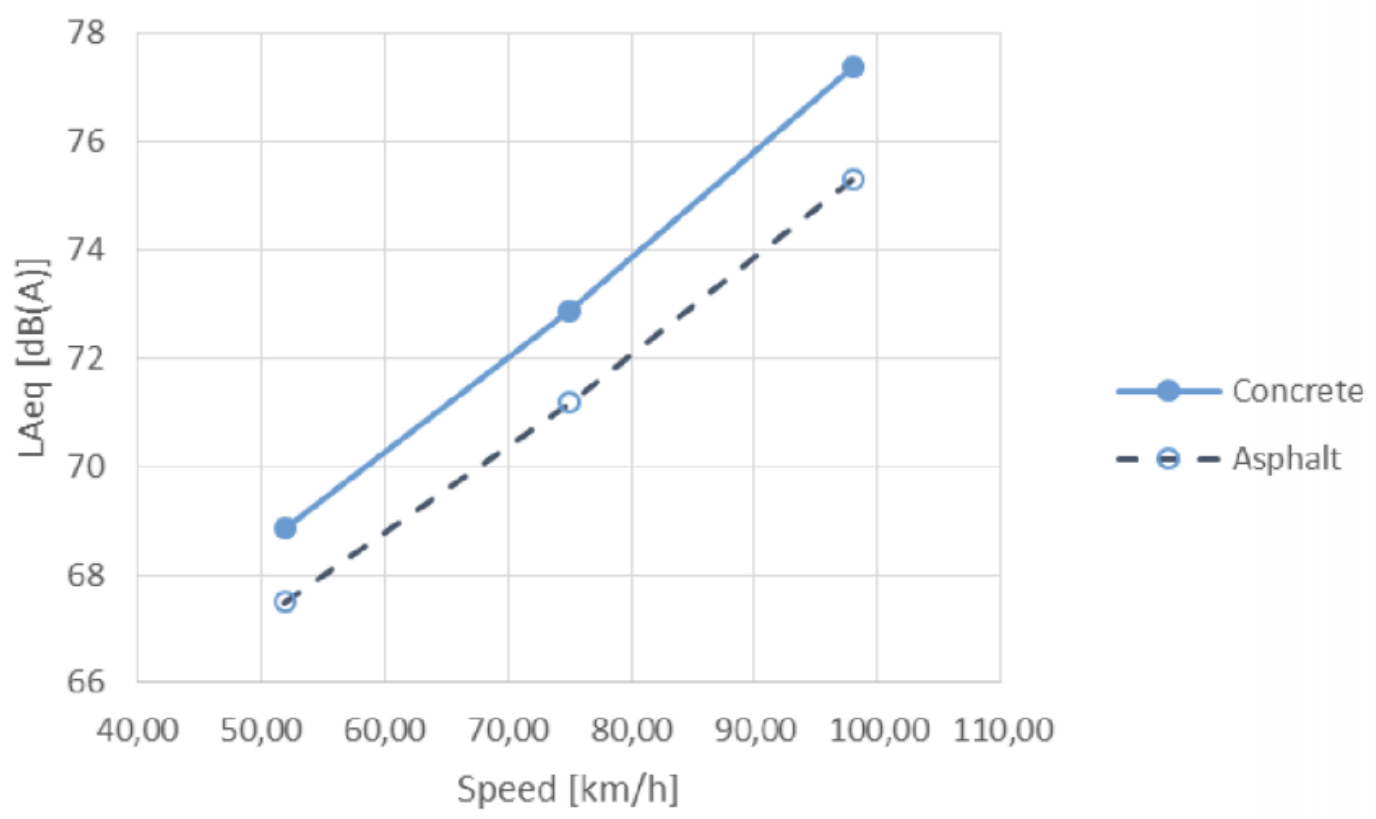

Figure 8:

the best choice. A decision maker will have to consider which impact or impacts they value the most to identify a clear winner.

Table 2: Results

\begin{tabular}{llll}
\hline & Do Nothing & Ramp Metering & No Off-Ramp \\
Delay & 3rd & 2nd & 1st \\
Emissions & Tied-1st & 2nd & Tied-1st \\
Noise Pollution & 3rd & 1st & 2nd \\
\hline
\end{tabular}

The rankings for each alternative are shown in table 10. From the table, we can be seen that there are trade-offs in costs and benefits between choosing different options. For example, the no off-ramp option has the least delay and emissions but has intermediate noise pollution value out of the alternatives. Assuming we value each impact equally, the no-off ramp alternative would likely be the best option and the do nothing alternative would be the worst. However, it should be noted that it is very difficult to quantitatively place a value on the cost emissions and noise pollution may have on the environment and human health.

\section{Conclusion}

We figure out that the most congested route is CA-24. The results would not change if different initial volumes were assumed. Some initial values would converge quicker than 
others, but we would always arrive at the same result after enough iterations.

In the queuing diagram, $\mathrm{D}(\mathrm{t})$ and $\mathrm{W}(\mathrm{t})$ have constant slopes of 2599.87 vehicles/hour and 6066.67 vehicles/hour respectively. $\mathrm{V}(\mathrm{t})$ has an initial slope of 3249.78 vehicles/hour until around 7:23 am when the slope changes to 520 vehicles/hour. And then we get the results as following:

- Duration of the congested period in the ramp:

From 6:02 to $7: 47$ (1:45 min)

- Total Delay: 758.369veh-hrs

- Maximum Delay: 0.333hrs

- Average Delay: 0.166675

- Total Cost: 1191.8 veh-hrs

- Maximum Cost: 0.333 hrs

- Average Cost: 0.264 hrs

Finding the equilibrium (Vickrey theory) we determine that the congested period lasts 1 hour and $45 \mathrm{~min}$, during this time the maximum delay experienced was $0.333 \mathrm{hrs}$ (20 min) with an average of $0.16 \mathrm{hrs}(9 \mathrm{~min})$. In terms of cost of time (considering the earliness and lateness factor), we found the average cost rise up to $0.264 \mathrm{hrs}(15 \mathrm{~min}$ ), however the maximum cost remains unchanged (0.333hrs).

To find the equilibrium among three route options we follow an iterative process based on the assumption that all the commuters choose their route option to minimize their travel time. One important result of this study the total volume (\# vehicles) in each route after the equilibrium has been reached (CA-4 1288 veh, CA-24 27783 veh, I-580 5366 veh).

After comparing all three alternatives we can see that the no off-ramp alternative produces the least delay, the do nothing and no off-ramp alternatives produce similar results for the least carbon dioxide emissions, and the ramp metering condition produces the least noise.

We conclude our analysis doing a cost benefit investigation. Considering time as the criteria we find that the No-Off ramp produces the least travel time (just considering delay), with a total delay of 546.7 veh-hr. The next point of comparison taken was the $\mathrm{CO}_{2}$ emission, this study was based in the EMFAC model, which relates the $\mathrm{CO}_{2}$ emission with the speed in queue in each alternative (do nothing, ramp metering, No Off-ramp). It was found that the No Off-ramp and the Do-nothing alternatives produces the least carbon dioxide emissions (around 33 tons).

Finally, we assume that the main variable that determines the noise pollution in the freeway section is the speed since we can expect that the distribution of the types of vehicles is the same among the three alternatives. In this case the optimum alternative is the ramp metering.

Since no alternative produces the least delay, emissions, and noise, we make the table as following:

There are trade-offs in costs and benefits between choosing different options. Assuming we value each impact equally, the no-off ramp alternative would likely be the best option and the do nothing alternative would be the worst. 
Table 3: ranking

\begin{tabular}{llll}
\hline & Do Nothing & Ramp Metering & No Off-Ramp \\
Delay & 3rd & 2nd & 1st \\
Emissions & Tied-1st & 2nd & Tied-1st \\
Noise Pollution & 3rd & 1st & 2nd \\
\hline
\end{tabular}

\section{References}

[1] D. Brownstone, M. Hansen, S. M. Madanat, Review of" bay area/california high-speed rail ridership and revenue forecasting study", Tech. rep. (2010).

[2] A. Bauranov, S. Parks, X. Jiang, J. Rakas, M. González, Quantifying the resilience of the us domestic aviation network during the covid-19 pandemic. front, Built Environ 7 (2021) 642295.

[3] M. Hansen, J. Tsao, S.-C. A. Huang, W. Wei, Empirical analysis of airport capacity enhancement impacts: A case study of dfw airport, University of California, Berkeley (1998).

[4] J. Li, X. Chen, X. Li, X. Guo, Evaluation of public transportation operation based on data envelopment analysis, Procedia-Social and Behavioral Sciences 96 (2013) 148-155.

[5] J. Everts, X. Jiang, Making sense of electrical vehicle discussions using sentiment analysis on closely related news and user comments, arXiv preprint arXiv:2112.12327 (2021).

[6] E. F. Morgul, H. Yang, A. Kurkcu, K. Ozbay, B. Bartin, C. Kamga, R. Salloum, Virtual sensors: Web-based real-time data collection methodology for transportation operation performance analysis, Transportation Research Record 2442 (1) (2014) 106-116.

[7] C. Chai, J. Lu, X. Jiang, X. Shi, Z. Zeng, An automated machine learning (automl) method for driving distraction detection based on lane-keeping performance, arXiv preprint arXiv:2103.08311 (2021).

[8] W. Huang, C. Yan, X. Jiang, Chemical and rheology evaluation on the field short-term aging of high content polymer modified asphalt, Tech. rep. (2019).

[9] K. Salimifard, H. Shahbandarzadeh, R. Raeesi, Green transportation and the role of operation research, in: Int. Conf. Traffic Transp. Eng.(ICTTE 2012), Vol. 26, 2012, pp. 74-79.

[10] Y.-y. Tseng, W. L. Yue, M. A. Taylor, et al., The role of transportation in logistics chain, Eastern Asia Society for Transportation Studies, 2005.

[11] J. Yin, T. Tang, L. Yang, J. Xun, Y. Huang, Z. Gao, Research and development of automatic train operation for railway transportation systems: A survey, Transportation Research Part C: Emerging Technologies 85 (2017) 548-572. 